This is the accepted version of the article:

Dhainaut J., Avci-Camur C., Troyano J., Legrand A., Canivet J., I maz I., Maspoch D., Reinsch H., Farrusseng D..

Systematic study of the impact of MOF densification into tablets on textural and mechanical properties.

CrystEngComm, (2017). 19. : 4211 - . 10.1039/c7ce00338b.

Available at: https://dx.doi.org/10.1039/c7ce00338b 


\title{
Systematic study of the impact of MOF densification into tablets on textural and mechanical properties $†$
}

\author{
J. Dhainaut, $\quad \ddagger^{* a}$ C. Avci-Camur, ${ }^{b}$ J. Troyano, ${ }^{b}$ A. Legrand, ${ }^{b}$ J. Canivet, ${ }^{a}$ \\ I. Imaz, ${ }^{b}$ D. Maspoch, ${ }^{b c}$ H. Reinsch ${ }^{d}$ and D. Farrusseng ${ }^{a}$
}

Four different metal-organic framework powders (UiO-66, UiO-66- $\mathrm{NH}_{2}$, UiO-67, and HKUST-1) were shaped into tablets. The effect of the applied pressure on porous properties, mechanical resistance and tablet bulk density is reported. We observe a linear relationship between densification and tensile strength for all four studied MOFs, with the slope being MOF-dependent. We also report conditions for improving significantly the volumetric uptake. Finally, we evaluated our tablets' stability over time in the presence of moisture.

\section{Introduction}

Less than 20 years after their discovery, commercial applications of metal-organic frameworks (MOFs) have been recently announced. ${ }^{1}$ We have also acknowledged in the very last few years the development of pilot lines, either batch processes under hydrothermal conditions, ${ }^{2}$ or continuous flow processes using water as solvent, ${ }^{3}$ and even solvent-free continuous processes. ${ }^{4}$ Thanks to the demonstration of these scalable pilot lines, the commercial applications of MOF will expand without any doubts.

Typically, synthesis processes lead to the production of MOFs as loose powders. However and except in very rare cases, their applications require bodies with a specific shape such as tablets, extrudates, granulates, monoliths or coatings to cite only the most common forms. For example, in catalysis, one of the usual forms is tablets which are obtained by pressing powders. Such bodies shall present mechanical resistance to attrition and hydrostatic pressure, while preserving as much as possible the original pore structure. BazerBachi et al. reported the catalytic conversion of vegetable oil using ZIF-8 tablets which were as active as loose ZIF-8 powders, paving the way for further application of shaped MOFs

\footnotetext{
a Université de Lyon, Université Claude Bernard Lyon 1, CNRS, IRCELYON - UMR 5256, 2 Avenue Albert Einstein, 69626 Villeurbanne Cedex, France.

E-mail: jeremy.dhainaut@univ-lille1.fr

${ }^{b}$ Catalan Institute of Nanoscience and Nanotechnology (ICN2), CSIC and the Barcelona Institute of Science and Technology, Campus UAB, Bellaterra, 08193 Barcelona, Spain

c ICREA, Pg. Lluís Companys 23, 08010 Barcelona, Spain

d MOFapps AS, c/o Smidig Regnskapsservice ANS, P. Box 24 Tåsen, 0801 Oslo, Norway † Electronic supplementary information (ESI) available: Data of all tablets, TG analyses, XRD. See DOI: 10.1039/c7ce00338b

$\ddagger$ Present address: Univ. Lille, CNRS, ENSCL, Centrale Lille, Univ. Artois, UMR 8181 - UCCS - Unité de Catalyse et de Chimie du Solide, F-59000 Lille, France.
}

in catalysis. ${ }^{5}$ On the other hand, for gas storage, it is of utmost importance to fill the storage tanks with the largest

amount of adsorbents. The void fraction within the adsorbent bed is a critical factor to control in the design of an adsorbent independently of its composition or structure. ${ }^{6,7}$ Therefore, MOFs should be pressed into solids of high density such as tablets in order to maximize their volumetric uptake.

Despite important efforts for the development of shaping processes suitable for MOFs, challenges linked to their intrinsic fragility remain. ${ }^{8}$ Limitations can be listed into three categories: (i) relatively low thermal stability with respect to oxides, impeding the use of classical shaping processes which are based on firing a binder-containing formulation after pelletization; ${ }^{9}$ (ii) relatively low chemical stability in the presence of solvents, including water, making extrusion processes not generalisable; ${ }^{10}$ and (iii) relatively low mechanical stability owing to their very high porosity and flexibility, leading to structural collapse when the applied pressure exceeds a given threshold. ${ }^{11}$

When taking these limitations into account, mild pelletisation by compression appears to be a practical solution for MOFs as it overcomes firing issues and the use of solvent while limiting the structural collapse. Two review articles dealing with MOF densification have made a quite exhaustive inventory in the field, which will not be discussed here. ${ }^{12}$ Nevertheless, it was concluded that tablets could be obtained by compressing binderless, pure MOF powders. ${ }^{13}$ The use of binders such as alumina, silica, graphite, or polyvinyl alcohol was also reported to reduce the structure degradation observed when even moderate strength is applied, but they also decrease the tablets' overall porosity proportionally.

The optimisation of MOF densification consists of finding a compromise between a gain in mechanical stability of their 
body and a loss of their initial properties. Ideally, the structural and textural properties of the MOF crystals should be preserved while the tablet density should be as high as possible. For example, Nandasiri et al. proposed an optimal tablet density of about $0.5 \mathrm{~g} \mathrm{~cm}^{-3}$ for MOF-5 and MIL-101IJCr) while maintaining their initial porosity. ${ }^{14}$ Similar binderless densification of other MOFs among the most studied, namely ZIF$8,{ }^{5} \mathrm{UiO}-66,{ }^{10}$ UiO-66-NH $2,{ }^{11,15}$ and HKUST- $1,{ }^{5,16}$ has already been reported. However, the tablet density increase comes with lower textural properties. This is especially the case of HKUST-1, which already collapses at modest applied pressure.

While those works pave the way for the further development of MOFs, parameters related to the shaping itself, such as the compression ramp speed and dwell time are rarely reported, although they can have tremendous importance for the final tablet properties. More surprisingly, very few mechanical tests were made although the tablets were usually reported as robust. As a result, the lack of standardization in densification studies prevents comparing mechanical stability and textural properties from one MOF to another. In addition, we may wonder whether conclusions of MOF structural stability drawn from densification data can be given when an allor-nothing compression type is applied.

Herein, we report a systematic study based on quantitative descriptors for the densification of UiO-66, UiO-67, UiO-66$\mathrm{NH}_{2}$ and HKUST-1 using a R\&D tableting machine. We measured the impact of the compression step on the textural properties (namely the specific surface area and the micropore volume), the bulk density and the mechanical resistance of the resulting tablets. In contrast to the general statement claiming that HKUST-1 can hardly be densified, we show here that robust tablets of HKUST-1 can be obtained without significant degradation of its microporous structure. In addition, we have investigated the use of a low content of expanded natural graphite (ENG) as a dried binder. Finally, we investigated the stability of our tablets after four months in the presence of moisture.

\section{Experimental section}

\subsection{Synthesis of MOF powders}

UiO-66 was prepared based on a protocol proposed by Kim et al. ${ }^{17} \mathrm{~A}$ DMF solution with a molar composition of zirconium chloride : terephthalic acid : hydrochloric acid $=1: 1: 1$ was heated at $120{ }^{\circ} \mathrm{C}$ for $24 \mathrm{~h}$ under stirring. The solid product was recovered by filtration and washed twice with DMF. The remaining solvent entrapped inside the powder was exchanged first with ethanol and then with acetone, using Soxhlet extraction. Finally, UiO-66 was activated under vacuum at $150{ }^{\circ} \mathrm{C}$ for $12 \mathrm{~h}$.

UiO-67 was prepared based on a protocol proposed by Shearer et al. ${ }^{18}$ A DMF solution with a molar composition of zirconium chloride : 4,4'-biphenyldicarboxylic acid : water : benzoic acid $=1: 1: 1.3: 5$ was heated at $120{ }^{\circ} \mathrm{C}$ for $24 \mathrm{~h}$ under stirring. The solid product was recovered by centrifuga- tion and washed three times with DMF, three times with ethanol, and three times with acetone. Finally, UiO-67 was activated under vacuum at $60{ }^{\circ} \mathrm{C}$ for $12 \mathrm{~h}$.

UiO-66- $\mathrm{NH}_{2}$ was synthesized using a spray-drying continuous flow method. ${ }^{19}$ A precursor suspension of $0.22 \mathrm{M} \mathrm{Z \textrm {ZOCl } _ { 2 }}$ $.8 \mathrm{H}_{2} \mathrm{O}$ and $0.20 \mathrm{M}$ 2-aminoterephthalic acid in a $24 \mathrm{~mL}$ mixture of water and acetic acid $(1: 1)$ was injected into a coil flow reactor at a feed rate of $2.4 \mathrm{~mL} \mathrm{~min}^{-1}$ and at a temperature of 90 ${ }^{\circ} \mathrm{C}$. The residence time inside the coil flow reactor was $63 \mathrm{~s}$. The resulting pre-heated solution was then spray-dried in a Mini Spray Dryer B-290 (Büchi Labortechnik) at a flow rate of $336 \mathrm{ml} \mathrm{min}^{-1}$ and an inlet air temperature of $150{ }^{\circ} \mathrm{C}$, using a spray cap with a $0.5 \mathrm{~mm}$-diameter hole, affording a yellow powder. This powder was then redispersed in ethanol and collected by centrifugation. The two-step washing process was repeated with acetone. The final product was dried for $12 \mathrm{~h}$ at $60{ }^{\circ} \mathrm{C}$ in air. This dried powder was finally activated under vacuum at $200{ }^{\circ} \mathrm{C}$ for $6 \mathrm{~h}$ (temperature slope of $4{ }^{\circ} \mathrm{C} \mathrm{min}^{-1}$ ).

HKUST-1 was prepared using the spray-drying technology. ${ }^{20}$ A solution of $\left.0.90 \mathrm{M} \mathrm{CulJNO}\right)_{2} \cdot 2.5 \mathrm{H}_{2} \mathrm{O}$ and $0.60 \mathrm{M}$ 1,3,5-benzenetricarboxylic acid in $135 \mathrm{~mL}$ of a mixture of DMF, ethanol and water $(1: 1: 1)$ was spray-dried in a Mini Spray Dryer B-290 (Büchi Labortechnik) at a feed rate of 4.5

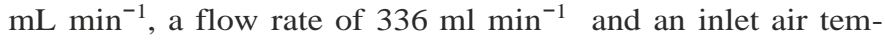
perature of $180{ }^{\circ} \mathrm{C}$, using a spray cap with a $0.5 \mathrm{~mm}$-diameter hole, affording a blue powder. This powder was then redispersed in ethanol and precipitated by centrifugation. The two-step washing process was repeated with acetone. The final product was dried for $12 \mathrm{~h}$ at $60{ }^{\circ} \mathrm{C}$ in air. This dried powder was finally activated under vacuum at $150{ }^{\circ} \mathrm{C}$ for $12 \mathrm{~h}$ (temperature slope of $4{ }^{\circ} \mathrm{C} \mathrm{min}^{-1}$ ).

\subsection{Densification method and body characterization}

A Medel'Pharm STYL'ONE Evolution tableting instrument was used for the shaping purpose. Prior to compression, the powders were deagglomerated by grinding. The die was then filled at constant volume with $150 \mathrm{mg}$ (UiO-67), $200 \mathrm{mg}$ (UiO-66) or $300 \mathrm{mg}$ (HKUST-1, UiO-66- $\mathrm{NH}_{2}$ ) of the MOF powder. A punch diameter of $1.128 \mathrm{~cm}$ was used, allowing a tableting surface of $1.00 \mathrm{~cm}^{2}$. Asymmetric compression, where the penetration of the upper punch was fixed at $2 \mathrm{~mm}$, was used. From the moment the punches reached the powder filling height $\left(h_{0}\right)$, a compression time of about $4 \mathrm{~s}$ was applied to reach a specified thickness following a constant displacement rate. This displacement rate is thickness-dependent. Then the compression was held for $400 \mathrm{~ms}$, before the punches went back to $\mathrm{h}_{0}$ within $4 \mathrm{~s}$. To avoid any residue on the internal die surface, some cellulose was pressed between two MOF tablets.

After shaping, physical parameters of MOF tablets were measured using a Pharmatron SmartTest 50. These parameters include the weight, thickness, and diameter, allowing to calculate the tablet bulk density. The same apparatus was also used to evaluate the tensile strength, by pressing the side of the tablets at a constant $1 \mathrm{~mm} \mathrm{~s}^{-1}$ displacement rate until a first load drop was detected. Such pressed tablets were usually 
split into two halves. The degree of densification is reported here as a percentage of the crystal density which is in theory the highest density that can be achieved for a crystal. Similarly, we express the specific surface area (SSA) of the tablet as a percentage of the initial powder. The SSA is a relevant quantitative indicator, as a loss of SSA after compression indicates a partial collapse of the porous structure. Alternatively, the micropore volume can be used equally as a quantitative descriptor of the degradation of the porous networks (see Table S1†).

The main advantage in using these indicators is that they are non dimensional, bounded between 0 and $100 \%$ and hence can be used to compare MOFs regardless of their intrinsic properties. We also provide a novel descriptor called volumetric capacity $\left(\mathrm{V}_{\text {cap. }}\right)$, which shall be relevant especially for gas storage applications. The positive effect of the shaping method is characterized by a higher volumetric capacity as compared to that of the initial powder. Moreover, the higher this descriptor is, the better the tablets shall perform for gas storage applications, until an optimum value is reached.

\subsection{Structure and porosity characterization}

Powder X-ray diffraction (PXRD) patterns were recorded on a Bruker D8 advance diffractometer using a $\mathrm{CuKa}$ radiation source and a Lynx-Eye detector. Nitrogen adsorption isotherms were measured at $77 \mathrm{~K}$ on a BELSORP-mini device from BEL Japan. The samples were outgassed at $150{ }^{\circ} \mathrm{C}$ overnight prior to measurement. Specific surface areas (SSAs) were determined using the BET method. The micropore volume ( $\mathrm{V}_{\text {micro. }}$ ) was evaluated from the adsorbed volume of $\mathrm{N}_{2}$ at $\mathrm{P} / \mathrm{P}_{0}$ $=0.3$. In order to calculate the bulk density of the tablets, dry masses were estimated from thermogravimetric (TG) analyses measured on a Mettler Toledo TGA-DSC1 using reconstituted air (Fig. S1 and Table S2†). Linker defects were also evaluated from TGA, assuming that the remaining weight at $800{ }^{\circ} \mathrm{C}$ corresponds to the metal oxide, namely $\mathrm{ZrO}_{2}$ (UiO-) or $\mathrm{CuO}$ (HKUST-1). This allows to determine directly the theoretical weight of a dehydroxylated MOF structure $\mathrm{Zr}_{6} \mathrm{O}_{6}$ IJlinker) 6 and $\mathrm{Cu}_{3} \mathrm{BTC}_{2}$, which is compared to the experimental remaining weight after solvent loss and dehydroxylation (taking metal oxidation into account). The weight difference is thus attributed to linker defects. This method doesn't take possible cluster defects into account. Powder bulk density, also called tap density, was determined by adding a known mass of deagglomerated MOF powder into a vial with a high height/diameter ratio. Then, the vial was repetitively hit on a hard surface until no more volume change could be observed - generally a few hundred times-, thus allowing to measure the packed bed dimensions. Scanning electron microscopy (SEM) was performed on a Hitachi S-4800.

\section{Results and discussion}

\subsection{Influence of compression on MOF tablet properties}

The impact of the densification step on the final tablet properties including density, SSA and tensile strength is presented in Fig. 1. First, we can see that the tap densities of MOF powders are very low with respect to their corresponding crystal density: it is only $14 \%$ for UiO-66, and the highest value is obtained for HKUST-1 with a relative density of $40 \%$. We can also note that while being isostructural to UiO-66, the UiO$66-\mathrm{NH}_{2}$ tap density reaches $32 \%$ of its crystal density. Here, the difference between these two isostructural MOFs is partly due to the synthesis protocol.

The MOF tablets were observed by SEM and the images are presented in the ESI. $\dagger$ Small and rounded crystals of HKUST-1 (Fig. S2†) and UiO-66- $\mathrm{NH}_{2}$ (Fig. S3†) are observed. No large agglomerate usually produced by spray-drying can be seen, ${ }^{19,20}$ meaning that grinding was successful at deagglomerating. UiO-67 prepared by a solvothermal method leads to micrometric crystals with a bipyramidal shape (Fig. $\mathrm{S} 4 \dagger$ ). The latter is typical of the isostructural $\mathrm{Zr}$-based series. While also being a part of this series, UiO-66 crystals are small and rounded (Fig. S5†). This is however in accordance with previous results. ${ }^{17}$ Moreover, the SEM images reveal that densification occurs more notably at the surface in contact with the punches.

For all studied MOFs, compression can yield tablets with twice higher density than their corresponding powders, while preserving more than $70 \%$ of their initial SSA. For instance HKUST-1, which has a reputation of being fragile, was densified to $75 \%$ of its crystal density while preserving $80 \%$ of its initial textural properties when pressed at $121 \mathrm{MPa}$. One may note that the densest random packing of spheres is about 64 to $74 \%$ of their three-dimensional volume. ${ }^{21}$ Surprisingly, we achieved here comparable values while limiting the loss of textural properties.

As expected, the higher the applied pressure, the higher the achieved densification. More interestingly, we observe a linear relationship between densification and tensile strength for all four studied MOFs below $80 \%$ relative density, with a slope being MOF-dependent. To the best of our knowledge, it is the first time that this behaviour is reported.

Obviously the densification level or the tensile strength cannot be enhanced by an ever increasing applied pressure without leading to porous structure collapse. When the highest density is sought, the loss of microporosity should be as reduced as possible; otherwise, it will affect the tablets' ultimate performances. For UiO-66, no loss of SSA has been observed until $40 \%$ relative density which is the highest level we have investigated as the aim was to reach a mechanical resistance high enough for further production of grains. This does not imply that a higher level of densification cannot be obtained without damaging the porous structure. For other studied MOFs, a modest but somehow linear decrease can be observed with increasing densification. Again, the decreasing rate is MOF-dependent.

Zr-based MOFs are known for their high mechanical and thermal stabilities owing to their 12-fold connected clusters in the three spatial directions. ${ }^{22,23}$ These stabilities can be compromised by the number of missing linkers per node usually about $2 ;^{24,25}$ however, the addition of monocarboxylic 

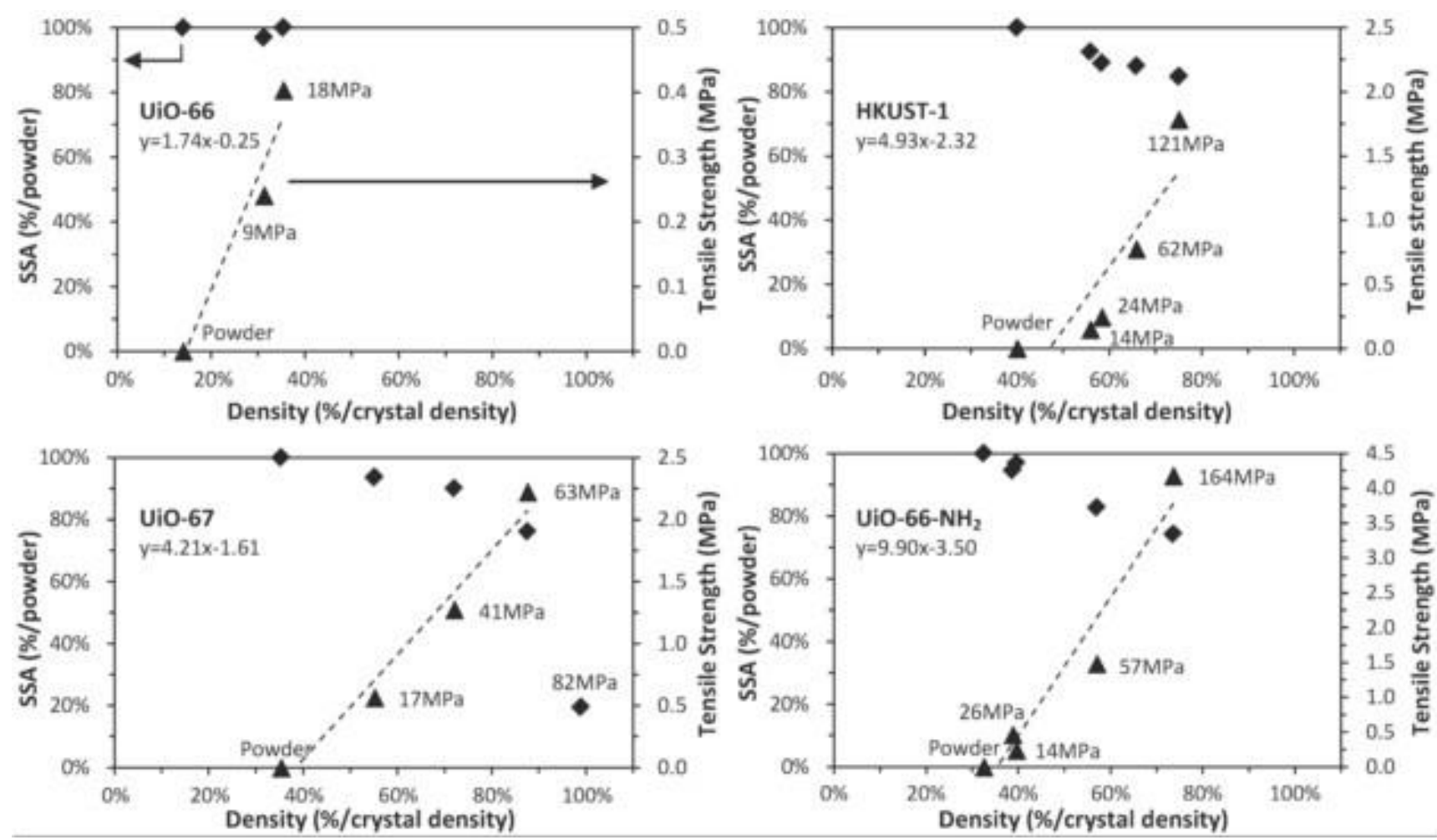

Fig. 1 Impact of the degree of compression on the textural properties (rhombus) and mechanical strength (triangle) for tablets of UiO-66, HKUST-1, UiO-67 and UiO-66- $\mathrm{NH}_{2}$. Applied pressures are indicated for each tablet, and the linear relationship tensile strength = flJrelative density) is reported. For UiO-67, the tensile strength was not measured above $63 \mathrm{MPa}$ as the structure collapsed.

acids during the synthesis of UiO-66 proved to enhance its stability. ${ }^{26}$ UiO-66 and UiO-67 powders prepared by solvothermal synthesis present $17 \%$ missing linkers per node ( 2 out of 12 ), which is comparable to the literature data. ${ }^{25,27}$ Although UiO-67 possesses the same topology as UiO-66 and the same degree of defects, its mechanical stability under compression is relatively modest as the porous structure collapses between 63 and $82 \mathrm{MPa}$ (SSA loss of about $80 \%$ ). This is in line with prior studies. ${ }^{25,28}$ Here, we can safely assume that the reason for this collapsing is due to the very high densification degree already reached, beyond $90 \%$, coupled with the very low flexibility of the framework. ${ }^{29}$

In summary, when MOF powders are pressed into tablets, up to a two-fold bulk density can be achieved at the expense of slightly reduced textural properties. For the production of MOF solids for catalysis, one may press a tablet only to reach an industrially-relevant tensile strength above $0.1 \mathrm{MPa},{ }^{30}$ thus limiting the impact of shaping on the gravimetric uptake.

In the case of gas storage and without precluding neither a specific gas nor application conditions, we can foresee that the densification level which would offer the greatest micropore volume per unit of volume should perform better assuming that the interaction is mainly van der Waals. We have calculated the volumetric capacity descriptor by multiplying the tablet microporous volume by their bulk density. This descriptor $\mathrm{V}_{\text {cap., }}$, presented in Table 1, is expressed in cubic centimetre of pore volume per cubic centimetre of tablet $\left(\mathrm{cm}^{3} / \mathrm{cm}^{3}\right)$. It can be pointed out that the micropore volume is correlated to the specific surface area and as a consequence, either one or the other can be used as descriptors (Fig. S6†). Detailed inputs for all tablets can be found in Table S2.† One may note that in most cases (HKUST-1, UiO-66, UiO-66- $\mathrm{NH}_{2}$ ), the "optimized" tablet is the one compressed at the highest pressure, meaning that the optimal pressure might not have been reached here and the volumetric capacity could still be increased. This is especially the case of UiO66, which is likely to resist compression pressures up to $2.500 \mathrm{MPa}$ with $50 \%$ of the initial SSA maintained. ${ }^{10}$ Nevertheless, a remarkable increase of tablet volumetric capacity can already be observed as compared to their powder counterparts, from 1.5 to 2.6 -fold. For example, the volumetric capacity of HKUST-1 can be increased from 0.24 to $0.36 \mathrm{~cm}^{3} /$ $\mathrm{cm}^{3}$. According to calculations made by Dailly et al., ${ }^{7}$ such densified HKUST-1 tablets could result in an outperforming adsorbed natural gas (ANG) adsorbent.

\subsection{Comparison with literature data}

Our results were further compared to the state-of-the-art literature in Fig. 2. For UiO-66 and UiO-66- $\mathrm{NH}_{2}$, we can see that the loss of SSA as a function of the applied pressure is well in line with the literature data. ${ }^{12,13}$

However, important discrepancies are observed in the case of HKUST-1. Herein, up to $85 \%$ of the original SSA of the 
Table 1 Textural properties and resulting volumetric capacity of optimized MOF tablets

\begin{tabular}{|c|c|c|c|c|c|c|c|c|}
\hline MOF & Form & $\begin{array}{l}\text { Applied pressure } \\
(\mathrm{MPa})\end{array}$ & $\begin{array}{l}\text { SSA } \\
\left(\mathrm{m}^{2} \mathrm{~g}^{-1}\right)\end{array}$ & $\begin{array}{l}V_{\text {micro. }} \\
\left(\mathrm{cm}^{3} \mathrm{~g}^{-1}\right)\end{array}$ & $\begin{array}{l}\text { Crystal density } \\
\left(\mathrm{g} \mathrm{cm}^{-3}\right)\end{array}$ & $\begin{array}{l}\text { Bulk density } \\
\left(\mathrm{g} \mathrm{cm}^{-3}\right)\end{array}$ & $\begin{array}{l}\text { Tensile strength } \\
(\mathrm{MPa})\end{array}$ & $\begin{array}{l}\mathrm{V}_{\text {cap. }} \\
\left(\mathrm{cm}^{3} / \mathrm{cm}^{3}\right)\end{array}$ \\
\hline \multirow[t]{2}{*}{ UiO-66 } & Powder & - & 1426 & 0.54 & \multirow[t]{2}{*}{1.21} & 0.17 & - & 0.09 \\
\hline & Tablet & 18 & 1459 & 0.54 & & 0.43 & 0.40 & 0.23 \\
\hline \multirow[t]{2}{*}{$\mathrm{UiO}-66-\mathrm{NH}_{2}$} & Powder & - & 839 & 0.34 & \multirow[t]{2}{*}{1.26} & 0.41 & - & 0.14 \\
\hline & Tablet & 164 & 625 & 0.25 & & 0.93 & 4.18 & 0.23 \\
\hline \multirow[t]{2}{*}{$\mathrm{UiO}-67$} & Powder & - & 2034 & 0.90 & \multirow[t]{2}{*}{0.71} & 0.25 & - & 0.23 \\
\hline & Tablet & 63 & 1549 & 0.70 & & 0.62 & 2.22 & 0.43 \\
\hline \multirow[t]{2}{*}{ HKUST-1 } & Powder & - & 1288 & 0.49 & \multirow[t]{2}{*}{1.20} & 0.48 & - & 0.24 \\
\hline & Tablet & 121 & 1091 & 0.40 & & 0.90 & 1.78 & 0.36 \\
\hline
\end{tabular}

powder was preserved at a moderate pressure of $120 \mathrm{MPa}$, while about $50 \%$ SSA loss was reported at lower pressures of 10 and $70 \mathrm{MPa}$ elsewhere. ${ }^{16}$ This could arise from several factors: (1) we used a relatively slow rate of pressure increase, which could allow HKUST-1 crystals to rearrange during compression; (2) as shown in Fig. 3, the solvent presence within the framework during compression allows maintaining its integrity, while in the literature, HKUST-1 powders are typically activated prior to compression; and (3) the presence of defects within the MOF structure is likely to lower its resistance during compression. Here, assuming that there is no cluster defect, the HKUST-1 powder used only presents $10 \%$ linker defects as shown in Table S2. $†$ The latter assumption can hardly be verified as it would require shaping exactly the same powders that were used elsewhere. One may note that in the literature, the mechanical resilience of zeolithic MOF single crystals was greatly improved using common solvents, namely ethanol and butanol. ${ }^{31}$

\subsection{Addition of expanded natural graphite as the binder for HKUST-1 powder}

Graphite is generally used as an additive for improving materials' thermal conductivity. ${ }^{32}$ Graphite was also applied as a mineral binder with UiO-66. ${ }^{33}$ Herein, up to $2 \mathrm{wt} \%$ expanded natural graphite was mixed with the HKUST-1 powder prior to compression, and its impact on both the textural proper-

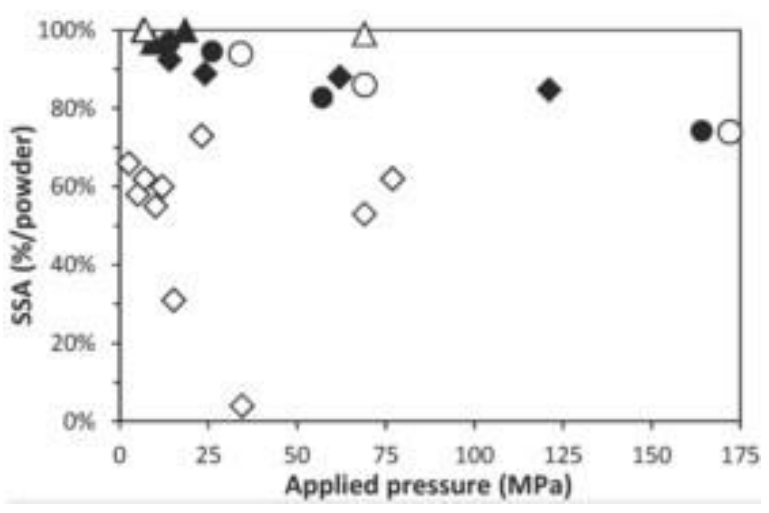

Fig. 2 SSA loss during compression observed in this work (black) and reported in the literature (white), using UiO-66 (triangle), UiO-66- $\mathrm{NH}_{2}$ (round) and HKUST-1 (rhombus) powders. ${ }^{10,15,16}$ ties and the tensile strength of the resulting tablet was investigated and presented in Fig. 4.

No measurable impact on the SSA could be observed at any of the applied pressure, while a minor enhancement of the tensile strength can be noted for $2 \mathrm{wt} \%$ ENG, almost independent of the applied pressure. This latter observation is particularly interesting for shaping MOFs at low pressure, thus maximizing the textural properties while reaching robustness high enough for applications.

\subsection{Overtime ageing of tablets in polyethylene bags}

After characterization, each tablet was sealed in a small polyethylene (PE) bag and left on a shelf under room conditions. It should be pointed out that PE bags are not impermeable. Indeed, their permeability to water vapor is $8.6 \times 10^{-4}$ $\mathrm{cm}^{3}$ IJSTP) $\mathrm{cm}^{-1} \mathrm{cmHg}^{-1}$ per day. ${ }^{34}$ The packaging in PE bags thus limits but does not prevent the water vapor exchange. The tablets with optimised volumetric capacity were then characterized again four months later in order to check their stability in the presence of moisture. The nitrogen adsorption isotherms of the different tablets are shown in Fig. 5 and their textural properties are summarized in Table 2. In the case of UiO-66 and $\mathrm{UiO}-66-\mathrm{NH}_{2}$, only a slight decrease of SSA and micropore volume was observed ( -9 to $-11 \%)$, while the PXRD was unchanged as shown in Fig. S7.†

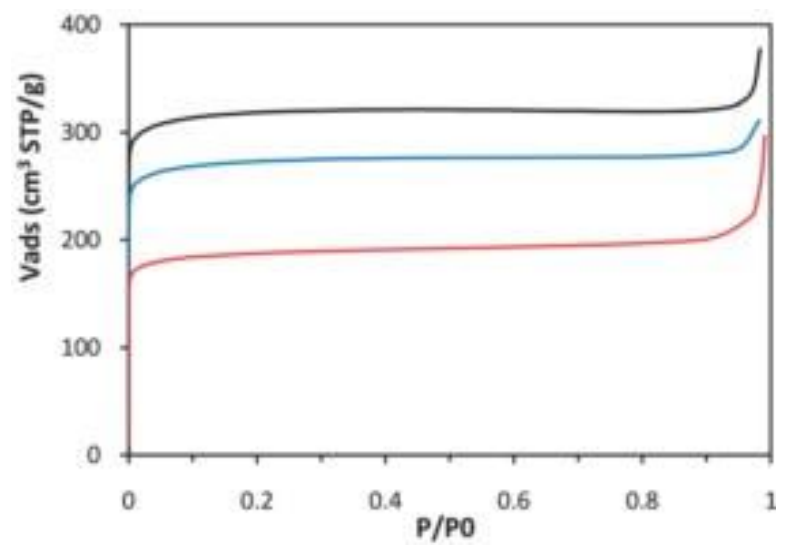

Fig. 3 Nitrogen adsorption isotherms of the as-made HKUST-1 powder (black/top) and HKUST-1 tablets pressed at $62 \mathrm{MPa}$ with the asmade powder (blue/middle) or with powder fully activated prior to compression (red/bottom). 


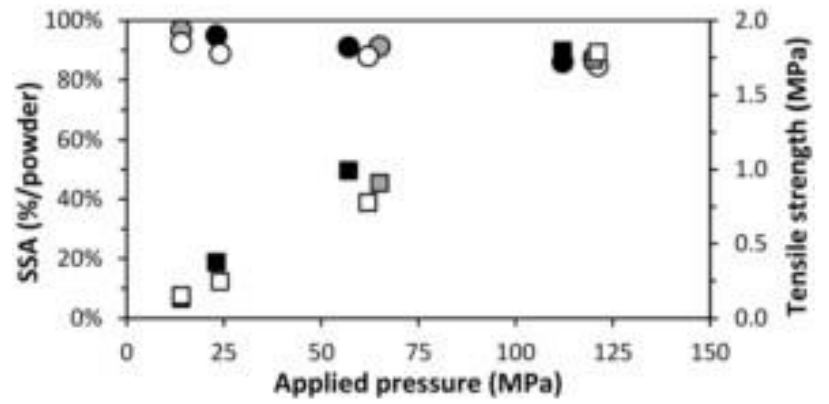

Fig. 4 Impact of compression on SSA (according to initial powder) and tensile strength of pure HKUST-1 tablets (white) or HKUST-1 mixed with $1 \mathrm{wt} \%$ graphite (grey) and $2 \mathrm{wt} \%$ graphite (black). Circles represent the SSA (according to initial powder), and squares represent the tensile strength.

In contrast, for UiO-67 tablets, $96 \%$ of their initial SSA was lost after 4 months. Notably, the PXRD patterns shown in Fig. S8† confirm a drastic loss of crystallinity. It was shown that UiO-67 is poorly stable under humid conditions. ${ }^{35}$ However, unlike what was published by DeCoste et al., ${ }^{35}$ no monoclinic zirconia diffraction peak could be observed; the degraded product can still be identified as pure UiO-67. This result confirms that $\mathrm{UiO}-67$ cannot be used in processes where even traces of water are present.

The HKUST-1 stability over time was extensively studied as it is one of the best candidates for gas storage and chromatography stationary phase. ${ }^{36}$ Todaro et al. investigated the HKUST-1 decomposition process with air moisture. ${ }^{37}$ They concluded that $\mathrm{Cu}-\mathrm{O}$ bond hydrolysis is driven by the accumulation of water molecules during exposure. This phenomenon is reversible for short exposure times, up to 20 days, following a simple vacuum treatment. For longer exposure
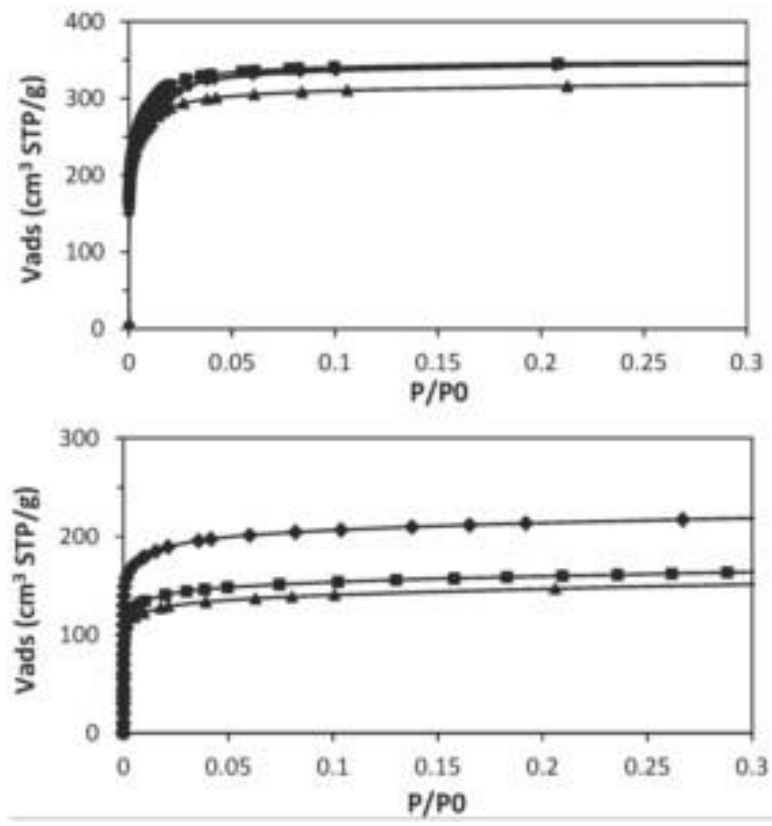

Table 2 Textural properties and resulting volumetric capacity of optimized MOF tablets after ageing for four months in the presence of moisture

\begin{tabular}{lcl}
\hline MOF & SSA $\left(\mathrm{m}^{2} \mathrm{~g}^{-1}\right)$ & $\mathrm{V}_{\text {micro. }}\left(\mathrm{cm}^{3} \mathrm{~g}^{-1}\right)$ \\
\hline UiO-66 & 1295 & 0.49 \\
UiO-66-NH & 570 & 0.24 \\
UiO-67 & 55 & 0.02 \\
HKUST-1 & 580 & 0.23
\end{tabular}

times, irreversible hydrolysis occurs, but up to $60 \%$ of the initial SSA is still preserved after 6 months. ${ }^{37}$ Interestingly, our HKUST-1 tablets aged similarly, with $53 \%$ of their initial SSA retained after 4 months. We can deduce that irreversible hydrolysis also occurred after tableting. Therefore, shaping MOF powders into dense tablets does not improve significantly their resistance toward air moisture. One can suppose that the formation of a dense crust of MOFs during tableting slows down the diffusion of moisture within the tablets at first, but does not prevent the degradation.

\section{Conclusions}

We present here a general methodology for comparing the impact of MOF powder compression on the textural and mechanical properties using non dimensional indicators. By compression, the tablet bulk density can be increased by 1.8 to 3.4-fold while the specific surface area decreased between 0 and $30 \%$ for all MOFs studied here. We report here for the first time that the mechanical stability, deduced from tensile strength tests, is proportional to the bulk density. Both are MOFdependent.
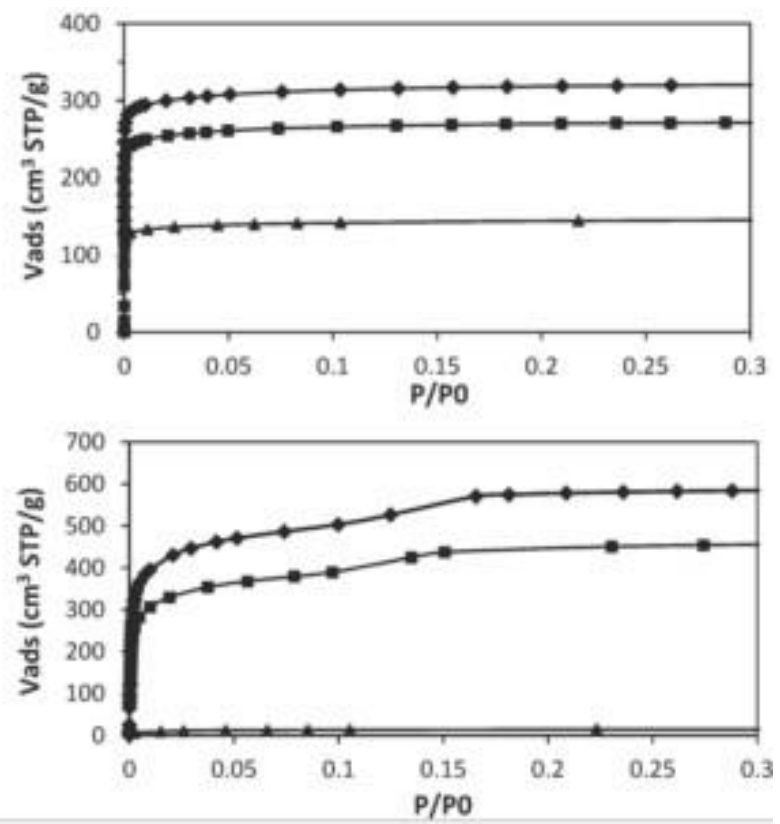

Fig. 5 Nitrogen adsorption isotherms measured for UiO-66 (top-left), UiO-66- $\mathrm{NH}_{2}$ (bottom-left), HKUST-1 (top-right) and UiO-67 (bottom-right): activated powders (rhombus), as-made tablets (square) and tablets aged for 4 months under standard room conditions (triangle). 
In contrast to all past densification studies carried out on HKUST-1, we demonstrated that robust tablets presenting only limited textural degradation can be obtained. We believe that it arises at least partly from our compression protocol, in which the compression rate and dwell time are thoroughly controlled. As a consequence, densification results obtained in an uncontrolled or poorly-controlled manner such as when using a manual press should be interpreted with care. Moreover, as reported in the case of zeolithic MOF single crystals, the presence of solvent traces within the pores of HKUST-1 seems beneficial during compression.

We underline here that depending on the final application, namely catalysis or gas storage, a different strategy can be used. For catalysis, a mild pressure, usually around 20 $\mathrm{MPa}$, seems enough to produce tough and highly porous tablets that can then be grinded and sieved. The use of graphite up to $2 \mathrm{wt} \%$ can also be beneficial as it lowers the pressure required to reach certain robustness, thus maximizing the retained textural properties. For gas storage, the maximum volumetric capacity was obtained at the highest applied pressure, except for UiO-67 which collapses at $82 \mathrm{MPa}$. However, only UiO-66 and $\mathrm{UiO}-66-\mathrm{NH}_{2}$ proved to be stable overtime in the presence of moisture, making them highly attractive for further production and applications.

\section{Acknowledgements}

This work has been carried out within the ProDIA project that has received funding from the European Union's Horizon 2020 research and innovation programme under grant agreement No 685727. We would like to thank S. Nieto Bobadilla and T. Menard from Medel'Pharm (FR) for providing their expertise, D. Cot (IEM, FR) for the SEM images, and Coldway (FR) for providing enhanced natural graphite.

\section{References}

1 Editorial, Nat. Chem., 2016, 8, 987.

2 A. S. Munn, P. W. Dunne, S. V. Y. Tang and E. H. Lester, Chem. Commun., 2015, 51, 12811.

3 M. Rubio-Martinez, T. D. Hadley, M. P. Batten, K.-C. Carey, T. Barton, D. Marley, A. Mönch, K.-S. Lim and M. R. Hill, ChemSusChem, 2016, 9, 1; H. Reinsch, S. Waitschat, S. M. Chavan, K. P. Lilerud and N. Stock, Eur. J. Inorg. Chem., 2016, 27, 4490.

4 D. Crawford, J. Casaban, R. Haydon, N. Giri, T. McNally and S. L. James, Chem. Sci., 2015, 6, 1645.

5 D. Bazer-Bachi, L. Assié, V. Lecocq, B. Harbuzaru and V. Falk, Powder Technol., 2014, 255, 52.

6 H. C. Foley and A. Qajar, Ind. Eng. Chem. Res., 2014, 53, 19649.

7 M. Beckner and A. Dailly, Appl. Energy, 2015, 149, 69; M. Beckner and A. Dailly, Appl. Energy, 2016, 162, 506.

8 A. I. Spjelkavik, A. Sevilime, S. Divekar, T. Didriksen and R. Blom, Chem. - Eur. J., 2014, 20, 8973; B. Böhringer, R. Fischer, M. R. Lohe, M. Rose, S. Kaskel and P. Küsgens, MOF
Shaping and Immobilization, in Metal-Organic Frameworks: Applications from Catalysis to Gas Storage, ed. D. Farrusseng, Wiley-VCH Verlag GmbH \& Co. KGaA, Weinheim, Germany, 2011.

9 S. Mitchell, N.-L. Michels and J. Pérez-Ramírez, Chem. Soc. Rev., 2013, 42, 6094; G. T. Whiting, A. D. Chowdhury, R. Oord, P. Paalanen and B. M. Weckhuysen, Faraday Discuss., 2016, 188, 369.

10 P. S. Bárcia, D. Guimarães, P. A. P. Mendes, J. A. C. Silva, V. Guillerm, H. Chevreau, C. Serre and A. E. Rodrigues, Microporous Mesoporous Mater., 2011, 139, 67; G. W. Peterson, J. B. DeCoste, T. G. Glover, Y. Huang, H. Jasuja and K. S. Walton, Microporous Mesoporous Mater., 2013, 179, 48.

11 P. G. Yot, K. Yang, F. Ragon, V. Dmitriev, T. Devic, P. Horcajada, C. Serre and G. Maurin, Dalton Trans., 2016, 45, 4283.

12 F. Akhtar, L. Andersson, S. Ogunwumi, N. Hedin and L. Bergström, J. Eur. Ceram. Soc., 2014, 34, 1643; S. C. McKellar and S. A. Moggach, Acta Crystallogr., Sect. B: Struct. Sci., Cryst. Eng. Mater., 2015, B71, 587.

13 K. W. Chapman, G. J. Halder and P. J. Chupas, J. Am. Chem. Soc., 2009, 131, 17546; M. A. Moreira, J. C. Santos,

A. F. P. Ferreira, J. M. Loureiro, F. Ragon, P. Horcajda, K.-E. Shim, Y.-K. Hwang, U.-H. Lee, J.-S. Chang, C. Serre and A. E. Rodrigues, Langmuir, 2012, 28, 5715; D. Peralta, G. Chaplais, A. Simon-Masseron, K. Barthelet and G. D. Pirngruber, Ind. Eng. Chem. Res., 2012, 51, 4692; R. Zacharia, D. Cossement, L. Lafi and R. Chahine, J. Mater. Chem., 2010, 20, 2145.

14 M. I. Nandasiri, S. R. Jambovane, B. P. McGrail, H. T. Schaef and S. K. Nune, Coord. Chem. Rev., 2016, 311, 38.

15

G. W. Peterson, J. B. DeCoste, F. Fatollahi-Fard and D. K. Britt, Ind. Eng. Chem. Res., 2014, 53, 701.

16 J. Kim, S.-H. Kim, S.-T. Yang and W.-S. Ahn, Microporous Mesoporous Mater., 2012, 161, 48; J. Liu, P. K. Thallapally and D. Strachan, Langmuir, 2012, 28, 11584.

17 S.-N. Kim, Y.-R. Lee, S.-H. Hong, M.-S. Jang and W.-S. Ahn, Catal. Today, 2015, 245, 54.

18 G. C. Shearer, S. Forselv, S. Chavan, S. Bordiga, K. Mathisen, M. Bjørgen, S. Svelle and K.-P. Lillerud, Top. Catal., 2013, 56, 770 .

19 L. Garzón-Tovar, M. Cano-Sarabia, A. Carné-Sánchez, C. Carbonell, I. Imaz and D. Maspoch, React. Chem. Eng., 2016, 1, 533.

20 A. Carné-Sánchez, I. Imaz, M. Cano-Sarabia and D. Maspoch, Nat. Chem., 2013, 5, 203.

21 J. R. Parrish, Nature, 1961, 190, 800.

22 L. Valenzano, B. Civalleri, S. Chavan, S. Bordiga, M. H. Nilsen, S. Jakobsen, K. P. Lillerud and C. Lamberti, Chem. Mater., 2011, 23, 1700.

23 M. Kandiah, M. H. Nilsen, S. Usseglio, S. Jakobsen, U. Olsbye, M. Tilset, C. Larabi, E. A. Quadrelli, F. Bonino and K.-P. Lillerud, Chem. Mater., 2010, 22, 6632; Y. Bai, Y. Dou, L.-H. Xie, W. Rutledge, J.-R. Li and H.-C. Zhou, Chem. Soc. Rev., 2016, 45, 2327. 
24 G. C. Shearer, S. Chavan, J. Ethiraj, J. G. Vitillo, S. Svelle, U. Olsbye, C. Lamberti, S. Bordiga and K. P. Lillerud, Chem. Mater., 2014, 26, 4068.

25 S. M. J. Rogge, J. Wieme, L. Vanduyfhuys, S. Vandenbrande, G. Maurin, T. Verstraelen, M. Waroquier and V. Speybroeck, Chem. Mater., 2016, 28, 5721.

26 B. Van de Voorde, I. Stassen, B. Bueken, F. Vermoortele, D. De Vos, R. Ameloot, J.-C. Tan and T. D. Bennett, J. Mater. Chem. A, 2015, 3, 1737.

27 M. J. Katz, Z. J. Brown, Y. J. Colón, P. W. Siu, K. A. Scheidt, R. Q. Snurr, J. Hupp and O. Farha, Chem. Commun., 2013, 49, 9449.

28 H. Wu, T. Yildirim and W. Zhou, J. Phys. Chem. Lett., 2013, 4, 925.

29 C. L. Hobday, R. J. Marshall, C. F. Murphie, J. Sotelo, T. Richards, D. R. Allan, T. Düren, F.-X. Coudert, R. S. Forgan, C. A. Morrison and S. A. Moggach, Angew. Chem., Int. Ed., 2016, 55, 2401.
30 S. Yamadaya, M. Oba, T. Hasegawa, K. Ogawa and Y. Kotera, J. Catal., 1970, 19, 264.

31 T. D. Bennett, J. Sotelo, J.-C. Tan and S. A. Moggach, CrystEngComm, 2015, 17, 286.

32 D. Liu, J. J. Purewal, J. Yang, A. Sudik, S. Maurer, U. Mueller, J. Ni and D. J. Siegel, Int. J. Hydrogen Energy, 2012, 37, 6109.

33

M. A. Moreira, J. C. Santos, A. F. P. Ferreira, J. M. Loureiro, F. Ragon, P. Horcajda, K.-E. Shim, Y.-K. Hwang, U.-H. Lee, J.-S. Chang, C. Serre and A. E. Rodrigues, Langmuir, 2012, 28, 5715 .

34 R. L. Hamilton, Bell Labs Tech. J., 1967, 46, 391.

35 J. B. DeCoste, G. W. Peterson, H. Jasuja, T. G. Glover, Y.-G. Huang and K. S. Walton, J. Mater. Chem. A, 2013, 1, 5642.

36

M. S. Singh, N. R. Dhumal, H. J. Kim, J. Kiefer and J. A. Anderson, J. Phys. Chem. C, 2016, 120, 17323.

37 M. Todaro, G. Buscarino, L. Sciortino, A. Alessi, F. Messina, M. Taddei, M. Ranocchiari, M. Cannas and F. M. Gelardi, J. Phys. Chem. C, 2016, 120, 12879. 